Article

\title{
Wavelet Based Denoising for the Estimation of the State of Charge for Lithium-Ion Batteries
}

\author{
Xiao Wang ${ }^{1,2}$, Jun $\mathrm{Xu}^{1,2, *(1)}$ and Yunfei Zhao ${ }^{1,2}$ \\ 1 State Key Laboratory for Manufacturing Systems Engineering, Xi'an Jiaotong University, Xi'an 710049, \\ Shaanxi, China; 13319210790@163.com (X.W.); zyf19940621@stu.xjtu.edu.cn (Y.Z.) \\ 2 Shaanxi Key Laboratory of Intelligent Robots, School of Mechanical Engineering, Xi'an Jiaotong University, \\ Xi'an 710049, Shaanxi, China \\ * Correspondence: xujunx@xjtu.edu.cn; Tel./Fax: +86-29-8266-3870
}

Received: 31 December 2017; Accepted: 24 April 2018; Published: 4 May 2018

\begin{abstract}
In practical electric vehicle applications, the noise of original discharging/charging voltage (DCV) signals are inevitable, which comes from electromagnetic interference and the measurement noise of the sensors. To solve such problems, the Discrete Wavelet Transform (DWT) based state of charge (SOC) estimation method is proposed in this paper. Through a multi-resolution analysis, the original DCV signals with noise are decomposed into different frequency sub-bands. The desired de-noised DCV signals are then reconstructed by utilizing the inverse discrete wavelet transform, based on the sure rule. With the de-noised DCV signal, the SOC and the parameters are obtained using the adaptive extended Kalman Filter algorithm, and the adaptive forgetting factor recursive least square method. Simulation and experimental results show that the SOC estimation error is less than $1 \%$, which indicates an effective improvement in SOC estimation accuracy.
\end{abstract}

Keywords: discrete wavelet transform; denoising; state of charge (SOC); adaptive extended Kalman filter

\section{Introduction}

With the development of electric vehicles, Battery Management Systems (BMS) have been widely used to monitor information ensuring battery safety and reliability [1-4]. Such information could be the current, the voltage, the temperature, etc. Defined as the available capacity over the maximum available capacity, the State of Charge (SOC) is estimated accurately with these signals to improve the performance of the battery system, to protect the battery from over-charging and over-discharging, and to prolong the service life of the battery.

However, to obtain accurate SOC is very challenging because the SOC cannot be measured directly through sensors, and the battery system is strongly non-linear. To solve this problem, a lot of research has been carried out on SOC estimation methods [5,6]. The ampere-hour counting (Coulomb counting) method is simple and easy to implement, but the method needs to know the initial SOC, and suffers from accumulated errors associated with noise and measurement errors. The open-circuit voltage $(\mathrm{OCV})$ method is very accurate, but this method needs a long rest time to estimate the SOC, and thus cannot be used in real time. Currently, SOC estimation methods based on the non-linear Equivalent Circuit Model (ECM), such as Extended Kalman Filter (EKF) [7-10], Proportional Integral Observer [11-13], and so on [14], are the most popular solutions. As the foundation of the model-based SOC estimation method, ECM is usually composed of the OCV and the resistance capacitance ( $\mathrm{RC}$ ) networks, and it makes the electrochemical behavior of a lithium-ion battery easier to understand. In model-based SOC estimation, this behavior is considered as the estimated terminal voltage. When discharging/charging pulse currents are applied to the batteries, discharging/charging 
voltage (DCV) signals are measured. The model-based SOC estimation method tracks the error between the estimated terminal voltage based on the ECM and the actually measured DCV signal to estimate SOC $[15,16]$. However, due to harsh electromagnetic environment in electric vehicles, the measured current and voltage signals can be seriously polluted. It is inevitable, therefore, that an uncorrected battery voltage is measured and applied to the BMS. Although the model-based SOC estimation method is used, the accuracy of SOC estimation is still reduced. Therefore, in order to improve the precision of SOC estimation, noise should be removed from the DCV.

For non-stationary signals, Fourier analysis is not effective since it transforms the signal into the frequency domain (and the time information is lost). This deficiency of the Fourier analysis can be mitigated to some extent by analyzing a small section of the signal at a time, called windowing. This type of analysis, known as the short-time Fourier transform, however, has the drawback that the size of the time window is the same for all frequencies [17-19]. The discrete wavelet transform (DWT) has been widely considered as an effective mathematical function to analyze DCV signals with non-stationary and transient phenomena in accordance with scale and resolution $[20,21]$. On the other hand, the wavelet transform involves a varied time-frequency window, and can provide good localization property in both the frequency and time domain.

In addition, with the SOC, ambient temperature, the number of cycles and other factors, the parameters of ECM including impedance will change. Currently, model parameters are identified by fitting a large amount of data collected under laboratory conditions, and the value of model parameters is fixed, which cannot dynamically reflect the influence of current, SOC, temperature, self-discharge on the internal characteristics of the battery. Although the model-based SOC estimation method can compensate for some system errors by feedback, it cannot completely eliminate the SOC estimation error caused by the factors given above. Therefore, in order to improve the accuracy of SOC estimation and enhance the adaptability of the system, it is necessary to estimate the battery model parameters online, and update the battery model in real time.

In order to solve the above-mentioned problems, this paper introduces a new approach to the application of discrete wavelet transform (DWT) - based denoising of DCV signals. The DWT has been widely considered as an effective mathematical function to analyze DCV signals with non-stationary and transient phenomena in accordance with scale and resolution [22]. At the same time, the first-order RC equivalent circuit model is established, and the model parameters are identified in real time by the Adaptive Forgetting Factor Recursive Least Square (AFFRLS). The Adaptive Extended Kalman Filter (AEKF) with Busse's adaptive rule is used to estimate SOC with the de-noised DCV signal. The results indicate that the proposed method can effectively reduce the impact of noise and improve the accuracy of SOC estimation. The remainder of the paper is organized as follows: In Section 2, the basic introduction on the DWT-based MRA is provided. The experimental platform is built to acquire the original DCV signal of the Li-ion cell. The original DCV signal is processed by the proposed DWT based MRA and threshold denoising technique to get the denoised signals. In Section 3, the first-order RC equivalent circuit model is established, and the model parameters are identified by AFFRLS. In Section 4, the AEKF is utilized to estimate SOC with the de-noised DCV signal. In the final section, conclusions are drawn, and final remarks are given.

\section{DWT-Based Denoising of DCV Signals}

\subsection{DWT and the Multi-Resolution Analysis}

DWT as an ideal tool for time-frequency analysis of non-stationary signal processing has been extensively studied in new mathematical approaches [22]. The DWT of the signal can be defined as

$$
D W T(j, k)=\frac{1}{\sqrt{2^{j}}} \int_{-\infty}^{\infty} x_{t} \psi *\left(\frac{t-k 2^{j}}{2^{j}}\right) d t
$$


where $\psi(t)$ is called the mother wavelet function, ${ }^{*}$ is the complex conjugate, and $j(j \in R)$ is the scale parameter determining the oscillation frequency and the length of wavelet. $k(k \in R)$ is the translation parameter, which determines the position on the time axis of the wavelet function. The scale parameter and the translation parameter of DWT provide the basis for Multi-Resolution Analysis (MRA), which enable MRA to provide high temporal resolution at high frequencies, and high frequency resolution at low frequencies. DWT-based MRA completely decomposes $x_{t}$ into the low-frequency approximate signal and the high-frequency detailed signal. The approximate signal is composed of the scaling function $\phi_{j, k}(t)$ and the approximate coefficients $a_{j, k}$. The detailed signal is composed of the wavelet function $\psi_{j, k}(t)$ and the detailed coefficients $d_{j, k}$. The scaling function $\phi_{j, k}(t)$ is directly related to the low-pass filter coefficient and the wavelet function $\psi_{j, k}(t)$ is directly associated with the high-pass filter coefficient. The original signal $x_{t}$ may be expressed by $J$ layer of DWT as:

$$
x(t)=\sum_{k=0}^{2^{N-J}-1} a_{J, k} 2^{-J / 2} \phi\left(2^{-J} t-k\right)+\sum_{j=1}^{J} \sum_{k=0}^{2^{N-J}-1} d_{j, k} 2^{-j / 2} \psi\left(2^{-j} t-k\right)
$$

where $N$ is called the maximum layer of decomposition and $J$ is the current layer of decomposition.

\subsection{Experimental Platform and Processing of DCV Signal}

In order to obtain the original DCV signal, this paper sets up a battery test platform. The experiment uses NCR18650 lithium-ion batteries with an actual capacity of $1573 \mathrm{mAh}$, a nominal voltage of $3.7 \mathrm{~V}$, and a cut-off voltage of $2.8 \mathrm{~V}$. Figure 1 shows the experimental platform used in this work. It is comprised of a host computer-monitoring system, battery test equipment, and an experimental chamber with constant temperature and humidity. The host computer-monitoring system sets the operating conditions and sends the required load current to the battery test equipment. According to the received current data, the battery test equipment makes the batteries charge and discharge. Simultaneously, the voltage sensor collects data to send to the host computer-monitoring system. The noise of the original DCV signal comes from the electromagnetic interference of the battery test platform and the measurement error of the sensor. Before the experiment, batteries are fully charged. The batteries recursively discharge to the cut-off voltage, according to Urban Dynamometer Driving Schedule (UDDS), to obtain the original DCV signal, as shown in Figure 2.



Figure 1. Battery test platform.

There is not a theoretical standard at present to select wavelet basis functions. The wavelet basis function has its own characteristics in signal processing, and no wavelet basis function can obtain the optimal denoising effect for all kinds of signals. In general, Daubechies wavelet family is one of the common discrete wavelet families, which is often used to denoise, due to its characteristics of orthogonality and tight support. The DCV signal is decomposed into the low-frequency approximate 
signal $A_{n}$ and the high-frequency detailed signal $D_{n}$, using the order 4 Daubechies wavelet (dB4), with the iterative decomposition of 5 layers [23]. The corresponding wavelet coefficients are the approximate coefficient $c A_{n}$ and the detailed coefficients $c D_{n}$, respectively, where is number of layers $(1 \leq n \leq 5)$. The decomposed DCV signal can be reconstructed by the inverse discrete wavelet transform (IDWT) using the same low and high-pass filter coefficients. The decomposition and reconstruction process is shown in Figure 3.

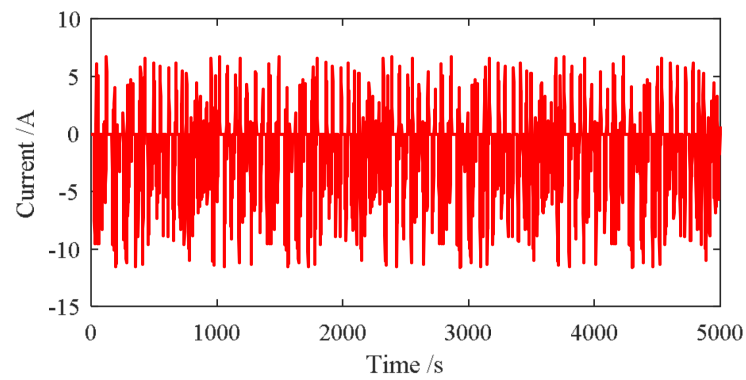

(a)

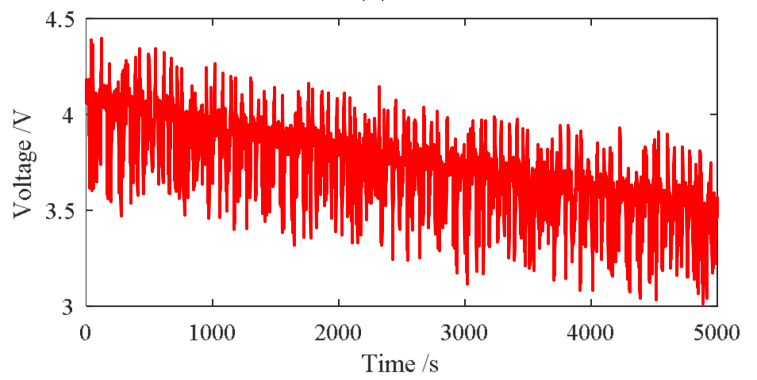

(b)

Figure 2. The measured signals: (a) the current of UDDS; (b) the original DCV signal collected by the battery test platform according to UDDS.

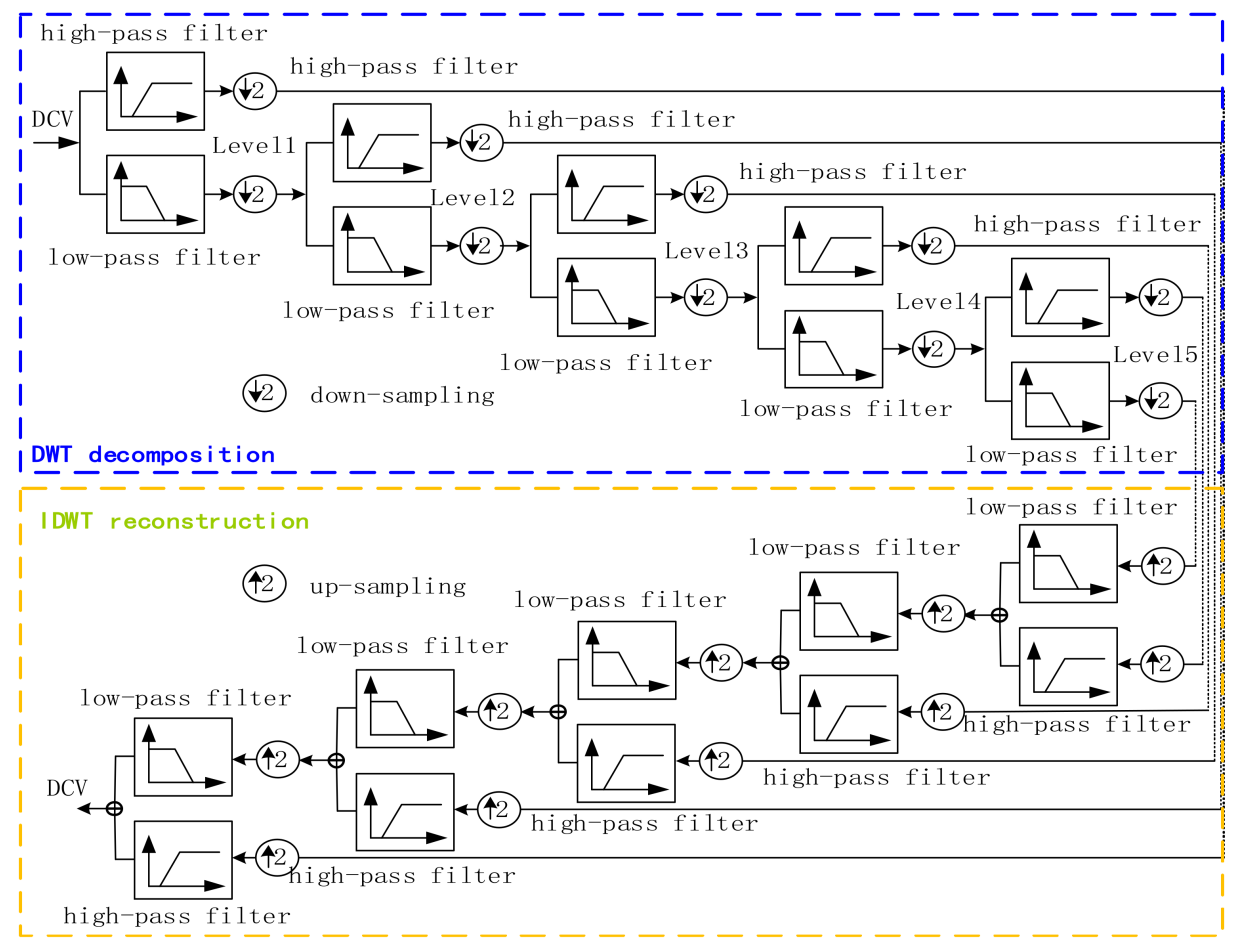

Figure 3. DWT-based MRA decomposition and reconstruction process of 5 layers. 


\subsection{The Denoised DCV Signal Based on the Thresholding-Based Denoising Rule}

In general, the original signal is expressed as the low-frequency signal or the smooth signal, and the noise is expressed as the high-frequency signal [24]. The original DCV signal with noise is decomposed to the low-frequency approximate signal and the high-frequency detailed signal. The noise signal is included in the high-frequency detailed wavelet coefficients $c D_{n}$. The amplitude and variance of high-frequency detailed component of the noise are decreasing with the increase of DWT decomposition levels. Therefore, in order to achieve the purpose of denoising, the high-frequency detailed wavelet coefficients $c D_{n}$ are processed by the threshold denoising rule, and the desired de-noised signal can be reconstructed by IDWT. The denoising process is shown in Figure 4.

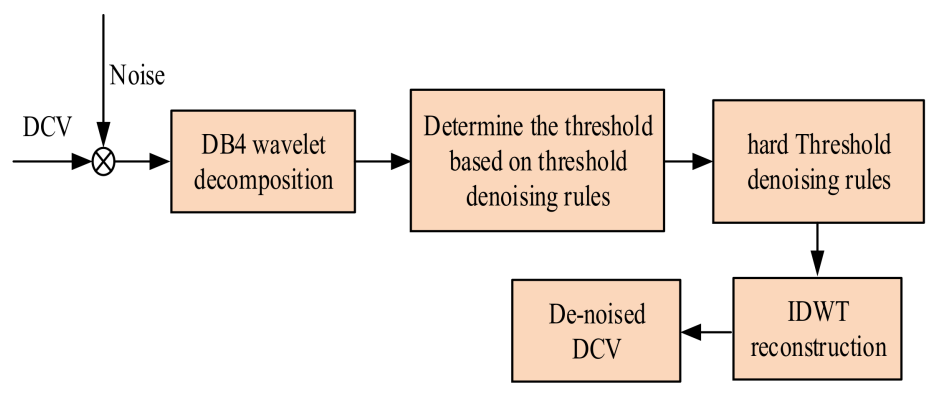

Figure 4. Hard threshold denoising process based on the threshold denoising rule.

A first-order RC ECM in Simulink is built to acquire the simulation DCV signal with the input of the experimental current signal noted above. The simulation DCV signal is considered as the noise-free signal. A band-limited white noise signal with noise power of 0.0003 is added into the simulation DCV signal to obtain the noisy simulation DCV signal with the signal-to-noise ratio (SNR) $25 \mathrm{~dB}$ [25]. Using a dB4 wavelet for the iterative decomposition of 5 layers decomposes the noisy simulation DCV signal, and the noise of the noisy simulation DCV signal is reduced by four hard threshold denoising rules based on the sqtwolong rule, sure rule, heursure rule and minimaxi rule, respectively [26]. The result of the denoising process is contrasted in Figure 5a. It can be seen from Figure 5 a that the noise reduction effect is better, and the curve-fitting effect with the noise-free simulation DCV signal curve is more ideal when based on the sure rule.

As shown in Table 1, comparing the SNR of the denoised DCV signal based on four hard-thresholding-based denoising rules, this paper selects the sure rule to deal with the noisy DCV signal. As shown in Figure 5b, noise in the noisy simulation DCV signal is better reduced.



(a)

Figure 5. Cont. 


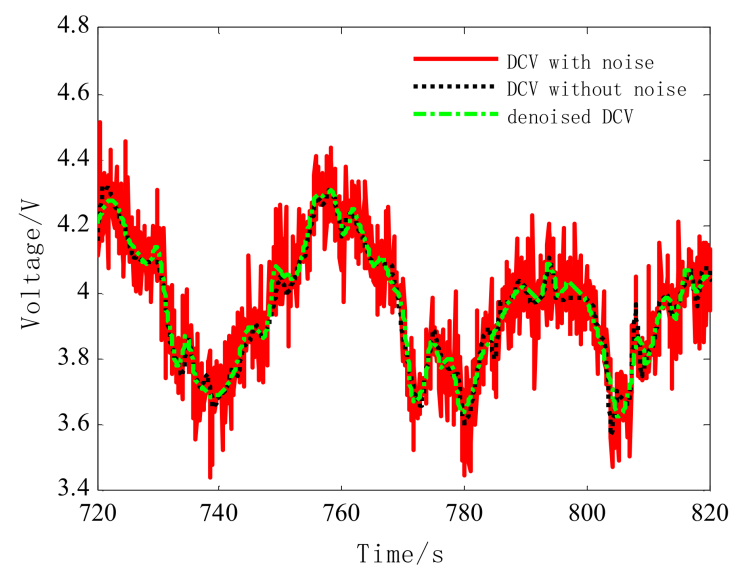

(b)

Figure 5. (a) The de-noised DCV signal processed by four hard threshold denoising rules; (b) The denoised DCV signal based on the sure rule.

Table 1. SNR of the denoised DCV signal.

\begin{tabular}{cc}
\hline Denoising Rules & SNR \\
\hline noisy simulation DCV signal & 25 \\
sqtwolong rule & 29.0315 \\
sure rule & 36.4833 \\
heursure rule & 29.6599 \\
minimaxi rule & 32.2239 \\
\hline
\end{tabular}

\section{The ECM of the Battery and the Parameter Identification Method}

\subsection{AFFRLS Algorithm}

A first-order RC ECM is adopted to simulate the dynamic characteristics of the lithium-ion battery in Figure 6. As shown in Figure $6, R_{t}$ is the ohm resistance, $R_{p}$ and $C_{p}$ are the polarization resistance and capacitance, respectively, $v_{p}$ is the polarization voltage, $v_{t}$ is the estimated terminal voltage, $E_{0}$ is the open circuit voltage. The relationship between SOC and $E_{0}$ is nonlinear. The battery has a rest time of an hour after SOC, decreasing by ten percent each time in the Hybrid Pulse Power Characteristic (HPPC) discharge experiment; at the same time, $E_{0}$ is measured. $E_{0}=a_{n} S O C+b_{n}$ is available by piecewise linear interpolation, and $a_{n}, b_{n}$ are shown in Table 2. Assuming the state variables $x=\left[x_{1}, x_{2}\right]^{T}=\left[v_{p}, S O C\right]^{T}$ and according to Kirchhoff's voltage law (KCL), the state equation is as:

$$
\left\{\begin{array}{l}
\dot{x}=A x+B u \\
y=C x+D u
\end{array}\right.
$$

where $A=\left[\begin{array}{cc}0 & 0 \\ 0 & -1 /\left(R_{p} C_{p}\right)\end{array}\right], B=\left[\begin{array}{c}1 / C_{n} \\ 1 / C_{p}\end{array}\right], C=\left[\begin{array}{cc}a_{n} & 1\end{array}\right], D=R_{t}, y=v_{t}-b_{n}, u=i$.

Discrete the Equation (3) by z transform [27] to obtain the Equation (4) as:

$$
v_{t}(k)=a_{1} \cdot v_{t}(k-1)-a_{2} \cdot v_{t}(k-2)+b_{0} \cdot i(k)-b_{1} \cdot i(k-1)+b_{2} \cdot i(k-2)
$$

where $a_{1}, a_{2}, b_{0}, b_{1}, b_{2}$ are the parameters to be estimated. 




Figure 6. The first-order RC equivalent circuit model.

Table 2. Parameter $a_{n}$ and $b_{n}$ of $E_{0}$-SOC piecewise linear interpolation.

\begin{tabular}{ccccccccccc}
\hline SOC & $\mathbf{0 . 0 - 0 . 1}$ & $\mathbf{0 . 1 - 0 . 2}$ & $\mathbf{0 . 2 - 0 . 3}$ & $\mathbf{0 . 3 - 0 . 4}$ & $\mathbf{0 . 4 - 0 . 5}$ & $\mathbf{0 . 5 - 0 . 6}$ & $\mathbf{0 . 6 - 0 . 7}$ & $\mathbf{0 . 7 - 0 . 8}$ & $\mathbf{0 . 8}-\mathbf{0 . 9}$ & $\mathbf{0 . 9 - 1 . 0}$ \\
\hline$a_{n}$ & 0.323 & 0.387 & 0.468 & 0.552 & 0.62 & 0.465 & 0.478 & 0.586 & 0.604 & 0.534 \\
$b_{n}$ & 3.565 & 3.558 & 3.542 & 3.517 & 3.49 & 3.567 & 3.559 & 3.484 & 3.469 & 3.532 \\
\hline
\end{tabular}

The basic idea of the recursive least squares algorithm is that the new estimate is equal to the sum of the old estimate and the correction value. The forgetting factor has a significant effect on the convergence rate and tracking performance. When $\lambda$ is equal to 1 , the errors at all times are considered without any forgetting function and the tracking capability is weak. It is insensitive to noise, and the parameter estimation error in steady state is small. When $\lambda$ is equal to 0 , only the errors of the current moment are considered and the tracking capability of time-varying parameters is strong, but it is more sensitive to noise. In a non-stationary environment, $\lambda$ should be small enough that the algorithm can quickly track the local trend of non-stationary signals. In a steady environment, it is hoped that $\lambda$ can be gradually increased to a suitable value to reduce the parameter estimation error [28]. Therefore, the AFFRLS is proposed to identify the parameters of the battery model and update the battery model in real time. The formula is as follows:

$$
\begin{gathered}
y(k)=\boldsymbol{\varphi}^{T}(k) \cdot \boldsymbol{\theta} \\
e(k)=y(k)-\boldsymbol{\varphi}^{T}(k) \cdot \hat{\boldsymbol{\theta}}(k-1) \\
\hat{\boldsymbol{\theta}}(k)=\hat{\boldsymbol{\theta}}(k-1)+\boldsymbol{K}(k) e(k) \\
\boldsymbol{K}(k)=\frac{\boldsymbol{P}(k-1) \boldsymbol{\varphi}(k)}{\lambda(k)+\boldsymbol{\varphi}^{T}(k) \boldsymbol{P}(k-1) \boldsymbol{\varphi}(k)} \\
\boldsymbol{P}(k)=\left(\boldsymbol{I}-\boldsymbol{K}(k) \boldsymbol{\varphi}^{T}(k)\right) \boldsymbol{P}(k-1) / \lambda \\
\lambda(k)=\lambda_{\min }+\left(1-\lambda_{\min }\right) 2^{L(k)} \\
L(k)=-r\left(\mu e^{2}\right)
\end{gathered}
$$

where $e(k)$ is $y(k)$ the estimated error, $K(k)$ is the algorithm gain, $\boldsymbol{\varphi}^{T}(k)=\left[v_{t}(k-1),-v_{t}(k-2)\right.$, $i(k),-i(k-1), i(k-2)], \boldsymbol{\theta}=\left[a_{1}, a_{2}, b_{0}, b_{1}, b_{2}\right]^{T}, \boldsymbol{P}(k)$ is the covariance matrix, $\lambda(k)$ is the forgetting factor, $\lambda_{\min }$ is the minimum value of the forgetting factor, taken as $0.57, \hat{\boldsymbol{\theta}}(0)$ and $\boldsymbol{P}(0)$ need to be pre-assigned based on experience and $r$ is a rounding function. According to the proposed algorithm, we can see that when $e(k)$ tends to infinity, the minimum value of the forgetting factor $\lambda(k)$ is obtained; $\lambda(k)$ is equal to 1 when $e(k)$ tends to zero. 


\subsection{Parameter Identification}

The simulation result is shown in Figure 7. The actual value of the battery parameter for comparison is obtained by the Hybrid Pulse Power Characteristic (HPPC) discharge experiment. As shown in Figure 7, the estimated value of $R_{t}, R_{p}$ and $C_{p}$ quickly converge to the actual value $0.041 \Omega, 0.011 \Omega$, and $2000 \mathrm{~F}$, and the fluctuation is small after stability.



(a)

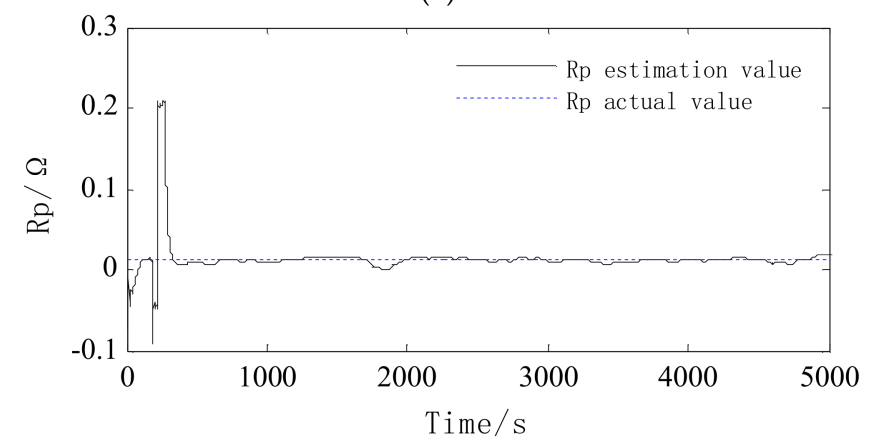

(b)



(c)

Figure 7. Parameter estimation and actual value: (a) ohm resistance; (b) polarization resistance; (c) polarization capacitance.

\section{The SOC Estimation Based on the AEKF Method}

\subsection{The AEKF Algorithm}

The Equation (3) is discretized to obtain a discrete battery model as follows:

$$
\left\{\begin{array}{c}
x_{k+1}=\left(A_{i}+E\right) x_{k}+B_{i} u_{k}+\omega_{k} \\
y=C x_{k}+D_{i} u_{k}+v_{k}
\end{array}\right.
$$


where $E$ is unit matrix. Matrix $A_{i}, B_{i}$ and $D_{i}$ are updated by $R_{t(\mathrm{i})}, R_{p(\mathrm{i})}$ and $C_{p(\mathrm{i})}$ obtained by AFFRLS. $A_{i}=\left[\begin{array}{cc}0 & 0 \\ 0 & -1 /\left(R_{p(\mathrm{i})} C_{p(\mathrm{i})}\right)\end{array}\right], B_{i}=\left[\begin{array}{c}1 / C_{n} \\ 1 / C_{p(\mathrm{i})}\end{array}\right], D_{i}=R_{t(\mathrm{i})} . \omega_{k}$ and $v_{k}$ are independent zero-mean white Gaussian noise, $\omega_{k}$ is the process noise with the covariance matrix $Q_{k}, v_{k}$ is the measurement noise with the covariance matrix $R_{k}$.

When using the EKF to implement SOC estimation, uncertain system noise may degrade the filter performance, and even cause divergence. Therefore, this paper utilizes EKF with Busse's adaptive rule [29] to adaptively update; the adaptive rule for the noise covariance update is defined as:

$$
\begin{gathered}
Q_{k}=Q_{k-1}+1 / L_{Q}\left(Q^{*}-Q_{k-1}\right) \\
Q^{*}=K_{k} e_{k} e_{k}^{T} K_{k}^{T}-P_{k}+(A+E) P_{k-1}(A+E)^{T} \\
R_{k}=R_{k-1}+1 / L_{R}\left(R^{*}-R_{k-1}\right) \\
R^{*}=e e^{T}-C_{k} P_{k-1} C_{k}^{T}
\end{gathered}
$$

where $e_{k}=y_{k}-\hat{y}_{k} . L_{Q}, L_{R}$ are the window size for process and measurement noise covariance respectively. $K_{k}$ is the Kalman gain, $P_{k}$ is the error covariance of the state $x_{k}$. In order to overcome the shortcomings of the poor real-time performance, this paper introduces a filter divergence judgment condition [30]. If the filter results are beyond the pre-set divergence conditions, we can only use the adaptive rule to start adaptive filtering projections, which can avoid divergence, effectively improving the stability and the real-time performance of the filter calculation. The divergence judgment condition is defined as

$$
e_{k}^{T} e_{k} \leq r T_{r}\left(\mathrm{E}\left(e_{k}^{T} e_{k}\right)\right)
$$

where $r$ is the adjustable coefficient, and $r \geq 1$. $T_{r}$ is the trace of the matrix. The remaining recursion process is as follows:

Time update:

$$
\begin{gathered}
\hat{x}_{k / k-1}=(A+E) \hat{x}_{k-1}+B u_{k-1} \\
P_{k / k-1}=(A+E) P_{k-1}(A+E)^{T}+Q_{k}
\end{gathered}
$$

Measurement update:

$$
\begin{gathered}
K_{k}=P_{k / k-1} C^{T}\left[\left(C P_{k / k-1} C^{T}+R_{k}\right)\right]^{-1} \\
\hat{x}_{k / k}=\hat{x}_{k / k-1}+K_{k} e_{k} \\
P_{k}=\left[I-K_{k} C\right] P_{k / k-1}\left[I-K_{k} C\right]^{T}+K_{k} R_{k} K_{k}^{T}
\end{gathered}
$$

\subsection{Simulation and Experimental Validation}

Using dB4 wavelet by the iterative decomposition of 5 layers decomposes the original DCV signal and the noise of the original DCV signal collected by the battery test platform; according to UDDS is reduced by sure rule. The result of the denoising process is contrasted in Figure 8. Obviously, it can be seen that when the current is zero, the voltage fluctuates abnormally for a long time. After using DWT based denoising, the noise can be filtered out. The new approach to the application of DWT based denoising of DCV signals has a good noise reduction effect. 


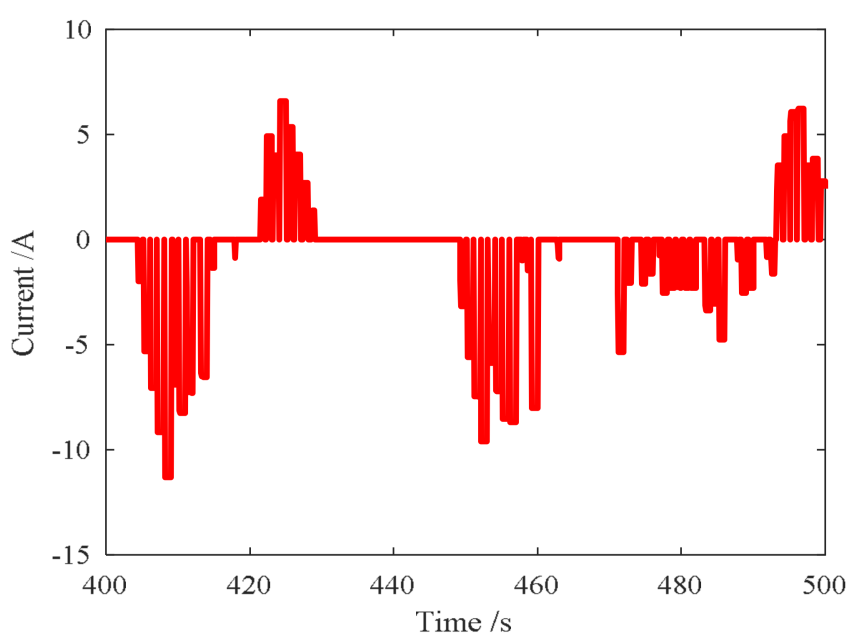

(a)

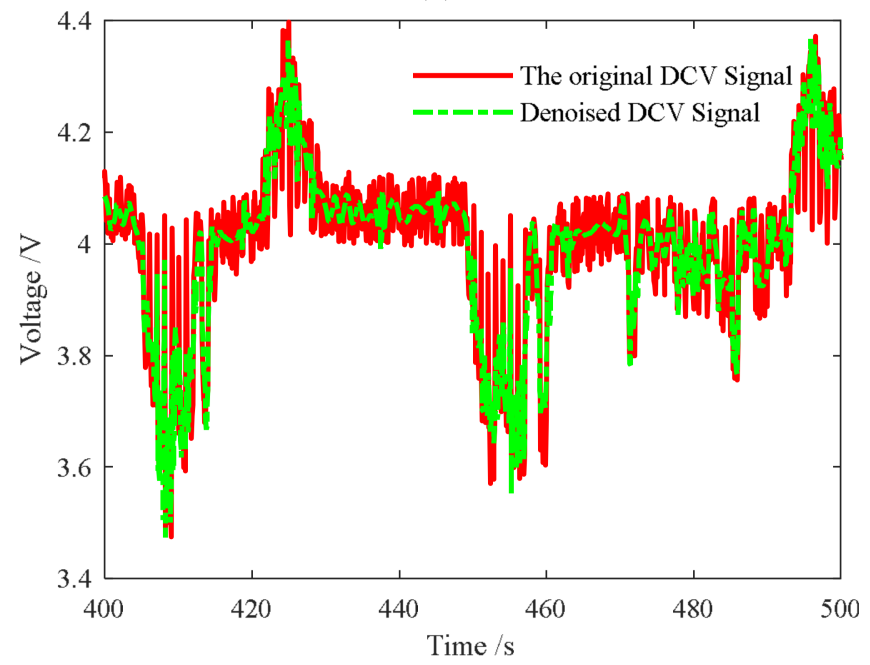

(b)

Figure 8. The measured and denoised signals: (a) the current of UDDS; (b) the original DCV signal collected by the battery test platform according to UDDS.

The noisy DCV signal and denoised DCV signal based on the sure rule are respectively used as a terminal voltage of the AEKF algorithm to estimate SOC. The SOC initial value is 0.8 . The simulation results are shown in Figure 9. It can be seen from Figure 9a when the SOC initialization error is $20 \%$, the AEKF-based SOC estimation of the denoised DCV signal rises to converge more rapidly to reference, based on the ampere-hour counting, than the DCV signal with noise. The AEKF-based SOC estimation of the denoised DCV signal has a smaller overshoot, and shows better robustness. Moreover, comparing with SOC estimation of the DCV signal with noise, it is closer to reference and has smaller fluctuation and higher precision. It can be seen from Figure $9 \mathrm{~b}$ that the AEKF-based SOC estimation error between the DCV signal with noise and the DCV without noise has larger fluctuations and exceeds 3\%. The AEKF-based SOC estimation of the denoised DCV signal substantially coincides with the DCV without noise, the SOC estimation error curve between them does not exceed $1 \%$, and fluctuation is smaller. Therefore, the DWT based denoising method proposed has a significant noise reduction effect and improves the accuracy of SOC estimation. 


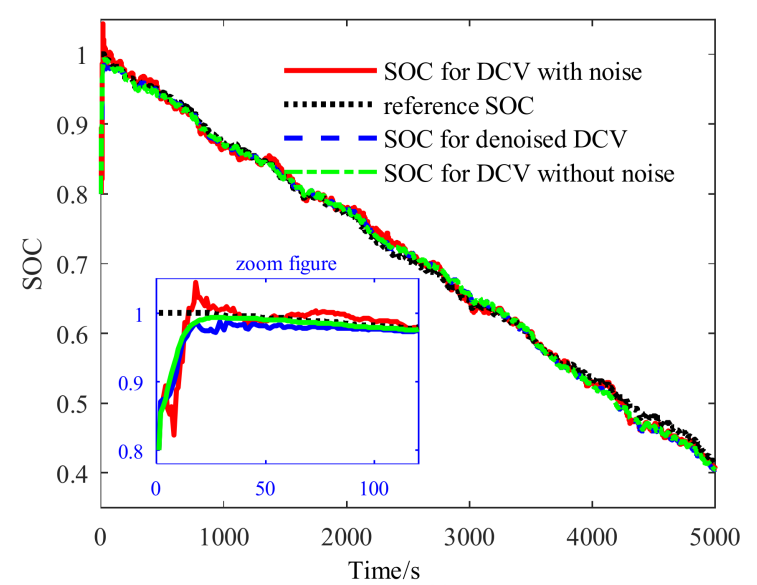

(a)

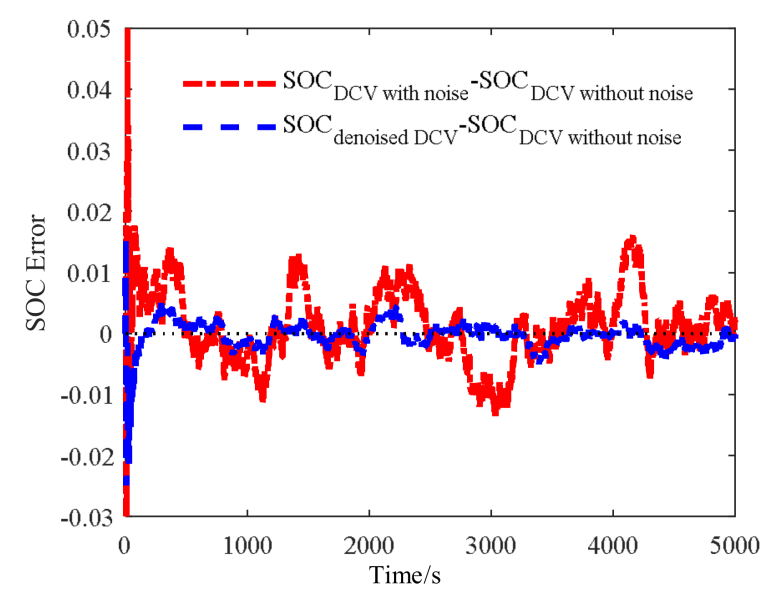

(b)

Figure 9. SOC estimation and error curve: (a) SOC estimation curve (initial value set to 0.8); (b) SOC estimation error curve.

\section{Conclusions}

The model-based SOC estimation method tracks the error between the estimated terminal voltage based on the ECM and the actually measured DCV signal to estimate SOC. An accurate and reliable DCV signal is absolutely necessary to improve the accuracy of SOC estimation. However, the actual measuring DCV signal will inevitably be mixed with noise. In addition, with the SOC, ambient temperature, the number of cycles and other factors changing, parameters of ECM including impedance will change a lot. Fixed parameters of ECM will reduce the accuracy of the model-based SOC estimation, and the model-based SOC estimation error will be huge and inevitable. In order to solve the above problems, this paper has proposed a new approach to taking advantage of the DWT-based denoising of DCV signals to obtain more accurate SOC estimation. By MRA, the original DCV signal obtained from the experiment was decomposed into different frequency sub-bands. By comparing the SNR of the de-noised DCV signal obtained by four threshold denoising rules, the hard threshold denoising rule based on the sure rule was selected to adjust the wavelet coefficients of the DWT, and realize the clear separation between the signal and the noise. The desired denoised DCV signal was reconstructed by taking the IDWT of the filtered detailed coefficients. On the other hand, a first-order RC equivalent circuit model was established, and the model parameters are identified in real time to update the battery model by the AFFRLS. Lastly, AEKF is used to estimate SOC with the denoised DCV signal, the DCV signal with noise and the DCV signal without noise. In order to verify the effectiveness of the proposed method, the SOC estimation based on AEKF has been compared with the ampere-hour 
counting method. The result shows that the SOC estimation error between the DCV signal with noise and the DCV without noise has larger fluctuations and exceeds $3 \%$. Whlie the SOC estimation of the denoised DCV signal substantially coincides with the DCV without noise, and the SOC estimation error between them does not exceed $1 \%$. In addition, the SOC estimation of the denoised DCV signal converges quickly to the reference based on the ampere-hour counting, and the fluctuation and error are smaller than the SOC estimation of the DCV signal with noise. In summary, the proposed method has a significant noise reduction effect of the measured DCV signal to increase the accuracy and reliability of the DCV signal, and the model parameters can be identified in real time to obtain more precise ECM, which improves the accuracy of SOC estimation.

Author Contributions: Jun Xu and Xiao Wang designed the overall algorithms and the simulations; Xiao Wang and Yunfei Zhao designed and performed the experiments; Xiao Wang and Jun Xu analyzed the data and wrote the paper.

Acknowledgments: This work was supported by the National Natural Science Foundation of China (Grant No. 51405374), the Postdoctoral Science Foundation of China (Grant No. 2014M560763), the Postdoctoral Science Special Foundation of China (Grant No. 2016T90904), the Fundamental Research Funds for the Central Universities and Postdoctoral Science Foundation of Shaanxi.

Conflicts of Interest: The authors declare no conflict of interest.

\section{References}

1. Meng, J.; Ricco, M.; Luo, G.; Swierczynski, M.; Stroe, D.I.; Stroe, A.I.; Teodorescu, R. An overview and comparison of online implementable soc estimation methods for lithium-ion battery. IEEE Trans. Ind. Appl. 2018, 54, 1583-1591. [CrossRef]

2. Xu, J.; Mi, C.C.; Cao, B.; Cao, J. A new method to estimate the state of charge of lithium-ion batteries based on the battery impedance model. J. Power Sources 2013, 233, 277-284. [CrossRef]

3. Xiong, R.; Zhang, Y.; He, H.; Zhou, X.; Pecht, M.G. A double-scale, particle-filtering, energy state prediction algorithm for lithium-ion batteries. IEEE Trans. Ind. Electron. 2017, 65, 1526-1538. [CrossRef]

4. Zheng, Y.; Ouyang, M.; Han, X.; Lu, L.; Li, J. Investigating the error sources of the online state of charge estimation methods for lithium-ion batteries in electric vehicles. J. Power Sources 2018, 377, 161-188. [CrossRef]

5. Zou, Z.; Xu, J.; Mi, C.; Cao, B.; Chen, Z. Evaluation of model based state of charge estimation methods for lithium-ion batteries. Energies 2014, 7, 5065-5082. [CrossRef]

6. Xu, J.; Cao, B. Battery Management System for Electric Drive Vehicles: Modelling, State Estimation and Balancing, 1st ed.; InTech: Rijeka, Croatia, 2015; pp. 87-113.

7. Plett, G.L. Sigma-point kalman filtering for battery management systems of lipb-based hev battery packs: Part 2: Simultaneous state and parameter estimation. J. Power Sources 2006, 161, 1369-1384. [CrossRef]

8. Plett, G.L. Extended kalman filtering for battery management systems of lipb-based hev battery packs: Part 2. Modeling and identification. J. Power Sources 2004, 134, 262-276. [CrossRef]

9. Xiong, R.; Sun, F.; Chen, Z.; He, H. A data-driven multi-scale extended kalman filtering based parameter and state estimation approach of lithium-ion olymer battery in electric vehicles. Appl. Energy 2014, 113, 463-476. [CrossRef]

10. Lim, K.; Bastawrous, H.A.; Duong, V.-H.; See, K.W.; Zhang, P.; Dou, S.X. Fading kalman filter-based real-time state of charge estimation in $\mathrm{LiFePO}_{4}$ battery-powered electric vehicles. Appl. Energy 2016, 169, 40-48. [CrossRef]

11. Xu, J.; Mi, C.C.; Cao, B.; Deng, J.; Chen, Z.; Li, S. The state of charge estimation of lithium-ion batteries based on a proportional-integral observer. IEEE Trans. Veh. Technol. 2014, 63, 1614-1621.

12. Zheng, L.; Zhang, L.; Zhu, J.; Wang, G.; Jiang, J. Co-estimation of state-of-charge, capacity and resistance for lithium-ion batteries based on a high-fidelity electrochemical model. Appl. Energy 2016, 180, 424-434. [CrossRef]

13. Xu, J.; Wang, J.; Li, S.; Cao, B. A method to simultaneously detect the current sensor fault and estimate the state of energy for batteries in electric vehicles. Sensors 2016, 16, 1328. [CrossRef] [PubMed]

14. Zou, C.; Manzie, C.; Nešić, D.; Kallapur, A.G. Multi-time-scale observer design for state-of-charge and state-of-health of a lithium-ion battery. J. Power Sources 2016, 335, 121-130. [CrossRef] 
15. Sepasi, S.; Ghorbani, R.; Liaw, B.Y. A novel on-board state-of-charge estimation method for aged Li-ion batteries based on model adaptive extended kalman filter. J. Power Sources 2014, 245, 337-344. [CrossRef]

16. Hu, X.; Sun, F.; Zou, Y. Estimation of state of charge of a lithium-ion battery pack for electric vehicles using an adaptive luenberger observer (vol 3,pg 1586, 2010). Energies 2011, 4, 2132. [CrossRef]

17. Bouzida, A.; Touhami, O.; Ibtiouen, R.; Belouchrani, A.; Fadel, M.; Rezzoug, A. Fault diagnosis in industrial induction machines through discrete wavelet transform. IEEE Trans. Ind. Electron. 2011, 58, 4385-4395. [CrossRef]

18. Kim, J.; Cho, B.H. An innovative approach for characteristic analysis and state-of-health diagnosis for a Li-ion cell based on the discrete wavelet transform. J. Power Sources 2014, 260, 115-130. [CrossRef]

19. Smith, C.M.; Nair, N.C. Comparing discrete wavelet transform (dwt) with discrete fourier transform (dft) implemented for digital relays. In Proceedings of the Power Engineering Conference, AUPEC 2009, Adelaide, Australia, 27-30 September 2009; pp. 1-6.

20. Muhit, A.A.; Islam, M.S.; Othman, M. Design and analysis of discrete wavelet transform(dwt) for image compression using vhdl. In Proceedings of the International Conference on Parallel and Distributed Processing Techniques and Applications, Pdpta 2005, Las Vegas, NV, USA, 27-30 June 2005; pp. 157-160.

21. Hwang, B.Y.; Jung, J.H.; Lee, J.M. Advanced sound source localization study using de-noising filter based on the discrete wavelet transform(dwt). J. Inst. Control 2015, 21, 1185-1192. [CrossRef]

22. Zhang, X.; Mi, C.C.; Masrur, A.; Daniszewski, D. Wavelet-transform-based power management of hybrid vehicles with multiple on-board energy sources including fuel cell, battery and ultracapacitor. J. Power Sources 2008, 185, 1533-1543. [CrossRef]

23. Maleknejad, K.; Yousefi, M.; Nouri, K. Computational methods for integrals involving functions and daubechies wavelets. Appl. Math. Comput. 2007, 189, 1828-1840. [CrossRef]

24. Kopsinis, Y.; McLaughlin, S. Development of emd-based denoising methods inspired by wavelet thresholding. IEEE Trans. Signal Process. 2009, 57, 1351-1362. [CrossRef]

25. Liu, C.-C.; Sun, T.-Y.; Tsai, S.-J.; Yu, Y.-H.; Hsieh, S.-T. Heuristic wavelet shrinkage for denoising. Appl. Soft Comput. 2011, 11, 256-264. [CrossRef]

26. Wei, W.; Zhang, Y.; Ren, G. Adaptive selection and simulation of optimal decomposition level in threshold de-noising algorithm based on wavelet transform. Chin. J. Sci. Instrum. 2009, 30, 526-530.

27. Rahimi-Eichi, H.; Chow, M.Y. Adaptive parameter identification and state-of-charge estimation of lithium-ion batteries. In Proceedings of the IECON 2012 - 38th Annual Conference on IEEE Industrial Electronics Society, Montreal, QC, Canada, 25-28 October 2012; pp. 4012-4017.

28. Paleologu, C.; Benesty, J.; Ciochina, S. A robust variable forgetting factor recursive least-squares algorithm for system identification. IEEE Signal Process. Lett. 2008, 15, 597-600. [CrossRef]

29. Yao, L.W.; Aziz, J.A.; Idris, N.R.N.; Alsofyani, I.M. Online battery modeling for state-of-charge estimation using extended kalman filter with busse's adaptive rule. In Proceedings of the Industrial Electronics Society, IECON 2015-Conference of the IEEE, Yokohama, Japan, 9-12 November 2015; pp. 004742-004747.

30. He, H.; Xiong, R.; Zhang, X.; Sun, F.; Fan, J. State-of-charge estimation of the lithium-ion battery using an adaptive extended kalman filter based on an improved thevenin model. IEEE Trans. Veh. Technol. 2011, 60, 1461-1469.

(C) 2018 by the authors. Licensee MDPI, Basel, Switzerland. This article is an open access article distributed under the terms and conditions of the Creative Commons Attribution (CC BY) license (http:/ / creativecommons.org/licenses/by/4.0/). 